\title{
Prediction of Depth of Sedation from Biological Signals Using Continuous Restricted Boltzmann Machine
}

\author{
Yeou-Jiunn Chen, Shih-Chung Chen, and Pei-Jarn Chen \\ Department of Electrical Engineering, Southern Taiwan University of Science and Technology, Tainan 710, Taiwan \\ Correspondence should be addressed to Pei-Jarn Chen; cpj@mail.stust.edu.tw
}

Received 18 August 2014; Accepted 1 September 2014; Published 11 September 2014

Academic Editor: Stephen D. Prior

Copyright (C) 2014 Yeou-Jiunn Chen et al. This is an open access article distributed under the Creative Commons Attribution License, which permits unrestricted use, distribution, and reproduction in any medium, provided the original work is properly cited.

\begin{abstract}
Proper anesthesia is very important for patients to get through surgery without pain and then avoid some other problems. By monitoring the depth of sedation for a patient, it could help a clinician to provide a suitable amount of anesthetic and other clinical treatment. In hospital, a patient is usually monitored by different types of biological systems. To predict the depth of sedation from biological signals is able to ease patient monitoring services. In this study, continuous restricted Boltzmann machines based neural network is proposed to predict the depth of sedation. The biological signals including heart rate, blood pressure, peripheral capillary oxygen saturation, and body weight are selected as analytic features. To improve the accuracy, the signals related to the state of anesthesia including fractional anesthetic concentration, end-tidal carbon dioxide, fraction inspiration carbon dioxide, and minimum alveolar concentration are also adopted in this study. Using minimizing contrastive divergence, a continuous restricted Boltzmann machine is trained and then used to predict the depth of sedation. The experimental results showed that the proposed approach outperforms feed-forward neural network and modular neural network. Besides, it would be able to ease patient monitoring services by using biological systems and promote healthcare quality.
\end{abstract}

\section{Introduction}

It is very important to help patients get through surgery without pain and avoid some other problems; thus clinicians need to provide proper anesthesia in surgery [1-4]. Under general anesthesia, memory and awareness are critical components of the depth of sedation (DOS). However, it needs additional monitoring equipment and sensors to predict the DOS. But, using this equipment and these sensors is uncomfortable for a patient. In clinical practice, a patient is usually monitored by different types of biological systems. Hence, it is important to predict the DOS from biological signals. It then can ease patient monitoring services and promote healthcare quality.

The assessment and interpretation of anesthesia are always based on patients' subjective reports, physiological monitors, and evaluation indices. Generally, electromyography, electrocardiography, blood pressure (BP), electroencephalogram, and peripheral capillary oxygen saturation $\left(\mathrm{SpO}_{2}\right)$ are monitored by physiological monitors. Bispectral index (BIS), entropy, auditory evoked potential, and surgical stress index are treated as evaluation indices. According to these data, an anesthetist is able to give an objective report of general anesthesia. But these approaches have their limits [5]. BIS is not real-time approach and it has a latency of 30 seconds or more [6]. The monitoring environment [7] for auditory evoked potential has a strict requirement. Entropy is sensitive to artifacts and noises caused by motions of body, such as eye movement and cough [8]. Besides, the equipment used in these approaches is very expensive and needs electrodes, which would be uncomfortable for a patient.

Recently, the researchers showed that the parasympathetic activity is significant relative to DOS $[9,10]$. Generally, the autonomic nervous system plays an important role in maintaining physiological activity. Therefore, the parasympathetic activity is usually monitored in clinical practice and can be represented as BP, heart rate (HR), sweat, and tear formation. Therefore, these physiological signals are used as a standard measure of autonomic reactions in clinical practice [11]. HR variability is closely related to autonomous 
nervous system, so the HR variability would vary remarkably between different narcotic states [12]. Because the HR is significant to monitor the depth of anesthesia, Li et al. applied HR variability to measure the degree of anesthesia [13]. In clinical practice, the strict safety limits are defined regarding respiratory rate, $\mathrm{HR}$, mean arterial blood pressure, $\mathrm{SpO}_{2}$, transcutaneous partial pressures of oxygen, and carbon dioxide [14]. Consequently, using these features would be feasible to improve the accuracy of estimation of DOS.

In the past decades, the restricted Boltzmann machine (RBM) had been widely used in many applications including dimensionality reduction, classification, collaborative filtering, feature learning, and topic modeling [15-21]. A RBM is a bipartite Markov random field wherein the input layer is associated with observed responses, and the output layer consists of hidden binary factors of variation [19]. The input layer is associated with observed responses and the output layer typically consists of hidden binary factors of variation. The standard type of RBM has binary-valued input and output layers. To extend the topics of applications, a continuous RBM (CRBM) is proposed to improve the modeling ability with both artificial and real continuous data by minimizing contrastive divergence (MCD) [22-24]. Therefore, the CRBM can be applied to different types of features and it could provide unsupervised feature learning and topic modeling. According to the advantages of CRBM, using CRBM as features learning and pattern classification would be able to preciously predict the DOS of a patient from biological signals.

In this study, CRBM based approach is proposed to predict the DOS from biological signals. To accurately predict the DOS, four features including $\mathrm{HR}, \mathrm{BP}$, and $\mathrm{SpO}_{2}$ are extracted to represent the biological signals. Because the status of anesthesia plays an important role in clinical anesthesia, integrating the features, which are relative to status of anesthesia, is able to accurately predict the DOS. Thus, four features including fractional anesthetic concentration (AA$\mathrm{FI}$ ), end-tidal carbon dioxide $\left(\mathrm{EtCO}_{2}\right)$, fraction inspiration carbon dioxide $\left(\mathrm{FiCO}_{2}\right)$, and minimum alveolar concentration (MAC) are adopted. Because these types of features are great relative to body weight $(\mathrm{BW})$, the $\mathrm{BW}$ should be also included. Finally, the CRBM whose parameters are trained by MCD is applied to predict DOS.

The rest of this paper is organized as follows. Section 2 describes the CRBM based approach for predicting DOS, including the feature extraction and CRBM. Section 3 presents the results of a series of experiments examining the performance of the proposed approach. In Section 4, the conclusion and recommendations for future research are presented.

\section{Method}

In this section, a CRBM based approach to predict DOS from biological signals is presented to improve performance beyond that of previously proposed methods. First, two types of features are extracted to represent the biological signals and the status of anesthesia. Second, the parameters of CRBM are estimated by using MCD training algorithm. Finally, the CRBM is used to predict the DOS. The procedure of proposed approach is detailed in the following subsections. Feature extraction is presented in Section 2.1. Then, the CRBM including continuous stochastic unit, diffusion network, and MCD training algorithm is detailed in Section 2.2.

2.1. Feature Extraction. Two types of features are selected in this study. The first type is related to patient's autonomic nervous system and the features are extracted from biological signals. These features are $\mathrm{HR}, \mathrm{BP}$, and $\mathrm{SpO}_{2}$, expressed as follows.

(i) Heart rate: a human's resting $\mathrm{HR}$ is determined by numerous and complex factors and then the anesthesia would usually cause a decrease in HR. HR is the speed of the heartbeat measured by the number of heartbeats per unit of time, typically beats per minute.

(ii) Blood pressure: $\mathrm{BP}$ is the pressure exerted by circulating blood upon the walls of blood vessels. Generally, it is measured in millimeters of mercury. During each heartbeat, blood pressure varies between maximum and minimum pressure.

(iii) Peripheral capillary oxygen saturation: $\mathrm{SpO}_{2}$ is an estimation of the oxygen saturation level.

The second type is related to the state of anesthesia and produced by an anesthesia monitor. These features are AAFI, $\mathrm{EtCO}_{2}$, and $\mathrm{FiCO}_{2}$, expressed as follows.

(i) Fractional anesthetic concentration: AA-FI is a chemical compound possessing general anesthetic properties that can be delivered via inhalation.

(ii) End-tidal carbon dioxide: $\mathrm{EtCO}_{2}$ is the level of carbon dioxide released at the end of expiration. A high $\mathrm{EtCO}_{2}$ reading in a patient with altered mental status or severe difficulty breathing may indicate hypoventilation and a possible need for the patient to be intubated. A low $\mathrm{EtCO}_{2}$ reading on patients may indicate hyperventilation, so it can quickly reveal a worsening trend in a patient's condition.

(iii) Fraction inspiration carbon dioxide: $\mathrm{FiCO}_{2}$ is the fraction or percentage of carbon dioxide in the space being measured.

(iv) Minimum alveolar concentration: MAC is the concentration of the vapor in the lungs that is needed to prevent movement in $50 \%$ of subjects in response to surgical stimulus.

Since BW greatly affects the effect of anesthesia, BW is always considered in clinical practice. Thus, the BW is selected as a feature in this study. Since the proposed model is based on neural network, the features of samples are normalized between 0 and 1 .

2.2. Continuous Restricted Boltzmann Machine. A CRBM, which is a stochastic neural network, consists of one visible layer and one hidden layer of stochastic neurons with 
interlayer connections [23]. An example of CRBM is shown in Figure 1. The probability generated from a visible neuron is proportional to the product of the probabilities that are generated by each of the hidden units acting along. The following subsections describe CRBM in detail.

2.2.1. Continuous Stochastic Unit. Let $v_{i}$ and $h_{j}$ be the $i$ th visible neuron and the $j$ th hidden neuron, respectively. Given the states of hidden neurons, the output of $v_{j}$ can be expressed as

$$
v_{i}=\varphi_{i}\left(\sum_{j} w_{j i} h_{j}+N_{i}(0, \sigma)\right)
$$

where $w_{i j}$ is the bidirectional weights connecting neuron $v_{i}$ and neuron $h_{j} . N_{j}$ is a zero-mean Gaussian noise with variance $\sigma^{2}$ and it constitutes a noise input component $n_{i}=$ $N_{i}(0, \sigma)$ according to the following probability distribution:

$$
p\left(n_{i}\right)=\frac{1}{\sqrt{2 \pi} \sigma} \exp \left(\frac{-n_{i}^{2}}{2 \sigma^{2}}\right) .
$$

$\varphi_{j}(\cdot)$ is the sigmoid function with asymptotes $\theta_{L}$ and $\theta_{H}$. Let the activation of $v_{j}$ be

$$
x_{i}=\sum_{j} w_{j i} h_{j}+N_{i}(0, \sigma) .
$$

Then, $\varphi_{j}(\cdot)$ can be rewritten as

$$
\varphi_{i}\left(x_{i}\right)=\theta_{L}+\left(\theta_{H}-\theta_{L}\right) \frac{1}{1+\exp \left(-a_{i} x_{i}\right)},
$$

where $a_{i}$ is the noise-control parameter and is used to control the slope of the sigmoid function. Therefore, it forms a continuous stochastic unit.

2.2.2. Diffusion Network. The network used in CRBM is a diffusion network, which consists of $n$ fully connected neurons with activation $x_{i}(t)$. Using a stochastic differential diffusion equation proposed by Movellan et al. [25], the diffusion network for the time evolution of the activation $x_{i}$ can be derived as

$$
d x_{i}(t)=\kappa_{i}\left(\sum_{j} w_{j i} \varphi_{i}\left(x_{i}(t)\right)-\rho_{i} x_{i}(t)\right) \cdot d t+\sigma \cdot d B_{i}(t),
$$

where $\kappa_{i}$ controls the changing rate of $x_{i}(t)$ and $\rho_{i}$ is a statedecay term. $d B_{i}(t)$ provides an additional random element in the network's behavior. Then, the discrete-time diffusion process for a finite time increment $\Delta t$ can be written as

$$
\begin{aligned}
x_{i}(t+\Delta t)= & x_{i}(t)+\kappa_{i} \sum_{j} w_{j i} \varphi_{j}\left(x_{i}(t)\right) \Delta t-\kappa_{i} \rho_{i} x_{i}(t) \Delta t \\
& +\sigma z_{i}(t) \sqrt{\Delta t},
\end{aligned}
$$

where $z_{i}(t)$ is a Gaussian random variable with zero mean and unit variance.

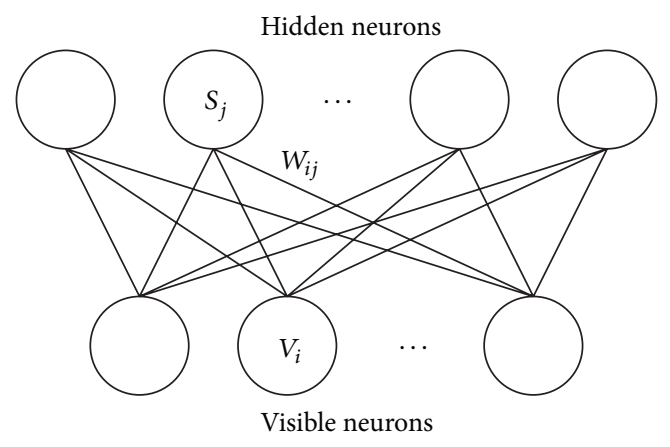

FIGURE 1: The architecture of CRBM.

Let $\Delta t=1 / \kappa_{i} \rho_{i}$ and $\sigma^{\prime}=\sigma \sqrt{\Delta t}$; then the term $x_{i}(t)$ can be canceled. Equation (6) can be rewritten as

$$
x_{i}(t+\Delta t)=\kappa_{i} \sum_{j} w_{j i} \varphi_{j}\left(x_{i}(t)\right) \Delta t+\sigma^{\prime} z_{i}(t) .
$$

If $W=\left\{w_{j i}\right\}$ is symmetric and $\kappa_{i}$ is a constant, (7) is equivalent to the total input of a CRBM as given by (1). When the CRBM is a symmetric restricted diffusion network, the weight update algorithm of the diffusion network can be used for training a CRBM.

2.2.3. MCD Training Algorithm for CRBM. A CRBM is trained by using an iterative training process that minimizes contrastive divergence [26]. In each training cycle, the training samples are sequentially used to modify the interlayer connections weights. Thus, the training rule for any parameter $\lambda_{i}$ in a diffusion network can be derived as

$$
\Delta \lambda_{i}=\left\langle v_{\lambda_{i}}\right\rangle_{0}-\left\langle v_{\lambda_{i}}\right\rangle_{\infty},
$$

where $\langle\cdot\rangle_{0}$ and $\langle\cdot\rangle_{\infty}$ are the exception values over the training data with visible states clamped. $v_{\lambda_{i}}$ is the system covariance which is the negative derivation energy function in diffusion network with respect to parameter $\lambda_{i}$. By minimizing contrastive divergence and avoiding the time-consuming process of full Gibbs's sampling to reach equilibrium, the $\left\langle v_{\lambda_{i}}\right\rangle_{\infty}$ can be treated as the exception value over one-step sample and then denoted by $\left\langle v_{\lambda_{i}}\right\rangle_{1}$. Thus, (8) can be rewritten as

$$
\Delta \widehat{\lambda}_{i}=\left\langle v_{\lambda_{i}}\right\rangle_{0}-\left\langle v_{\lambda_{i}}\right\rangle_{1} .
$$

Since the energy function of CRBM is analogous to the continuous Hopfield model [27], the energy function of CRBM can be derived as

$$
U=-\frac{1}{2} \sum_{i \neq j} w_{i j} v_{i} v_{j}+\sum_{j} \frac{1}{a_{j}} \int_{0}^{v_{j}} \varphi^{-1}(s) d s,
$$

where $\varphi(s)$ is $\varphi_{j}(s)$ with $a_{j}=1$. According to (9) and (10), the MCD training rules for weights $w_{i j}$ and noise-control parameters $a_{i}$ can be derived as

$$
\begin{gathered}
\Delta \widehat{w}_{i j}=\eta_{w}\left(\left\langle v_{i} v_{j}\right\rangle-\left\langle\widehat{v}_{i} \widehat{v}_{j}\right\rangle\right), \\
\Delta \widehat{a}_{i}=\eta_{a}\left(\frac{1}{a_{i}^{2}}\left\langle\int_{\widehat{v}_{i}}^{v_{i}} \varphi^{-1}(s) d s\right\rangle\right),
\end{gathered}
$$


where $\eta_{w}$ and $\eta_{a}$ are the learning rate. $\widehat{v}_{i}$ is the one-step sample state of neuron $i$ and $\langle\cdot\rangle$ is the mean over the training data. To simplify (12), the integral term is approximate as

$$
\int_{\widehat{v}_{i}}^{v_{i}} \varphi^{-1}(s) d s \cong\left(v_{i}+\widehat{v}_{i}\right)\left(v_{i}-\widehat{v}_{i}\right) .
$$

Therefore, (12) can be rewritten as

$$
\Delta \widehat{a}_{i}=\frac{\eta_{a}}{a_{i}^{2}}\left(\left\langle v_{i}^{2}\right\rangle-\left\langle\widehat{v}_{i}^{2}\right\rangle\right) .
$$

Finally, the training rules for CRBM in (11) and (14) can be simplified to use addition and multiplication operations.

\section{Experimental Results and Discussion}

In this study, the inhalational anesthetic was adopted and administered by anesthetists. The sevoflurane was used as the anesthetic gases and the biological signals were monitored by using Datex AS/5 anesthesia monitor. Therefore, a database including 6000 epochs was collected from 27 subjects (13 males and 14 females). 5000 and 1000 epochs were randomly selected as training and testing sets, respectively. Leaveone-out cross-validation was used to evaluate the proposed approach. The mean square error (MSE) was adopted to evaluate the performance of the proposed approach. Because the performance of neural network is greatly dependent on the initial weights connecting neurons, each approach was examined 20 times. The MSE were then used in the following experiments.

3.1. Experimental Results of CRBM in Different Formulas. In this subsection, the types of features and the number of neurons in hidden layer were examined. The maximum dimension of features is eight in this study and it is a low dimension for a neural network. Thus, the number of neurons in hidden layer for CRBM was tested from 1 to 100.

Besides, three types of features were considered according to the characteristics of features. The features for first type (denoted by BioFea) were related to the biological signals and they are $\mathrm{HR}, \mathrm{BP}, \mathrm{SpO}_{2}$, and $\mathrm{BW}$. The features for second type (denoted by AneFea) were related to the state of anesthesia and they are $\mathrm{AA}-\mathrm{FI}, \mathrm{EtCO}_{2}, \mathrm{FiCO}_{2}, \mathrm{MAC}$, and $\mathrm{BW}$. Because, these features were greatly affected by BW, the BW was considered in these two types. Finally, the features for third type were all the eight features and were denoted by ComFea.

The experimental results for three types of features were shown in Figure 2. The best performances for biological signals and the state of anesthesia were 0.0524 (with 4 neurons of hidden layer) and 0.0599 (with 69 neurons of hidden layer), respectively. It is clear that the performance of biological signals is better than that of the state of anesthesia. Besides, the number of neurons in hidden layer of CRBM is also quite small and it would greatly improve the computational efficiency. Thus, the biological signals are useful to predict the DOS.

Using ComFea, the MSE was 0.0504 when the number of neurons of hidden layer for CRBM was 6. It is clear

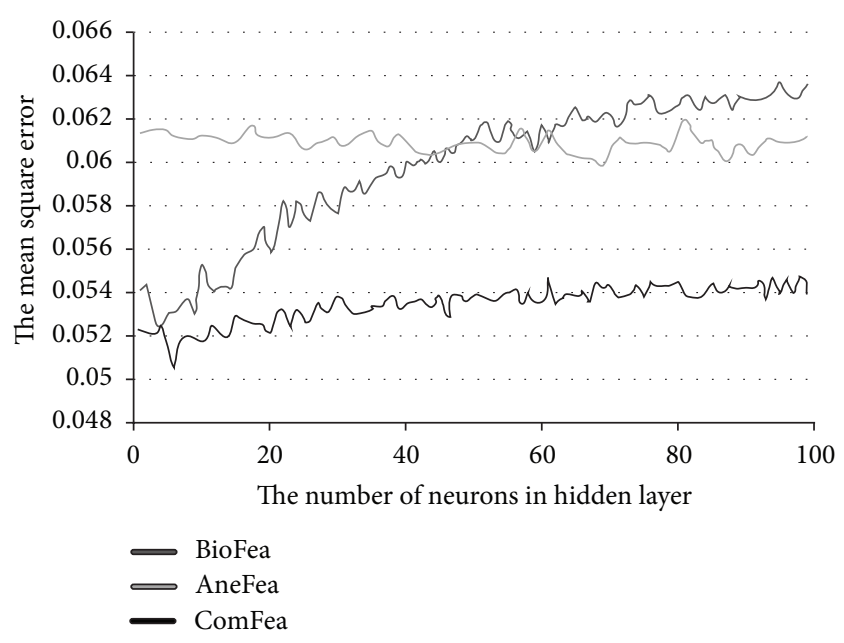

FIGURE 2: The experimental results of proposed approach using different types of features.

that the proposed approach using ComFea achieved the best performance. Therefore, the CRBM could effectively transform the features into a reasonable domain. It is also able to obtain a reasonable result in low dimension. Thus, the CRBM is very suitable in this topic. To avoid the effect of initial weights of a neural network, the proposed approaches were examined 20 times and then the experimental results of three types of features, which were expressed in maximum MSE, average MSE, minimum MSE, and number of neurons in hidden layer, were shown in Table 1 . We found that the minimum MSE of AneFea is larger than the maximum MSE of BioFea and ComFea. Therefore, the predictive ability of AneFea is lower than that of other types of features. Since the performance of BioFea is similar to that of ComFea, the proposed approach can be integrated with other biological systems. Then, it would effectively reduce the computational complex and increase the value of biological systems.

An example of predicted results was shown in Figure 3. The black line was the DOS for BIS, which was predicted from the signals of EEG. We could find that the variation of DOS estimated from BIS was larger than that of proposed approach (shown in red line). Therefore, the energy of EEG is noisesensitive and it would cause the result of DOS to be unstable. In clinical anesthesia, these noise-sensitive components can be ignored; thus the proposed approach provides reasonable results.

3.2. Comparison with Other Approaches. To compare the proposed approach, the feed-forward neural network (denoted by ANN) and modular neural network (MNN) were implemented as baseline system. Because the features of MNN should be classified into two groups, three grouping types were examined in this study. For the first grouping type of $\mathrm{MNN}$, the features including $\mathrm{HR}, \mathrm{BP}, \mathrm{SpO}_{2}$, and $\mathrm{EtCO}_{2}$ were selected as one group and the others were another group (denoted by $\mathrm{MN}-1$ ) [28]. For the second grouping type of MNN, the features, which were relative to the status of anesthesia (AA-FI, AA-ET, AA-MAC, and BW), were 
TABLE 1: The performance of proposed approach using different types of features.

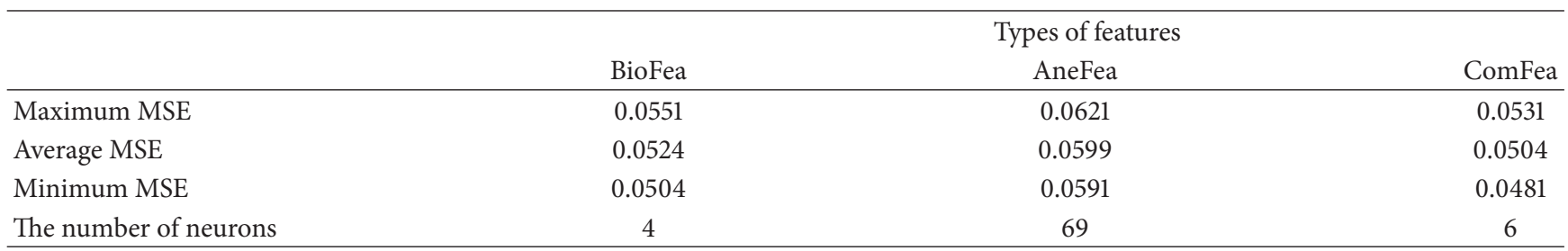

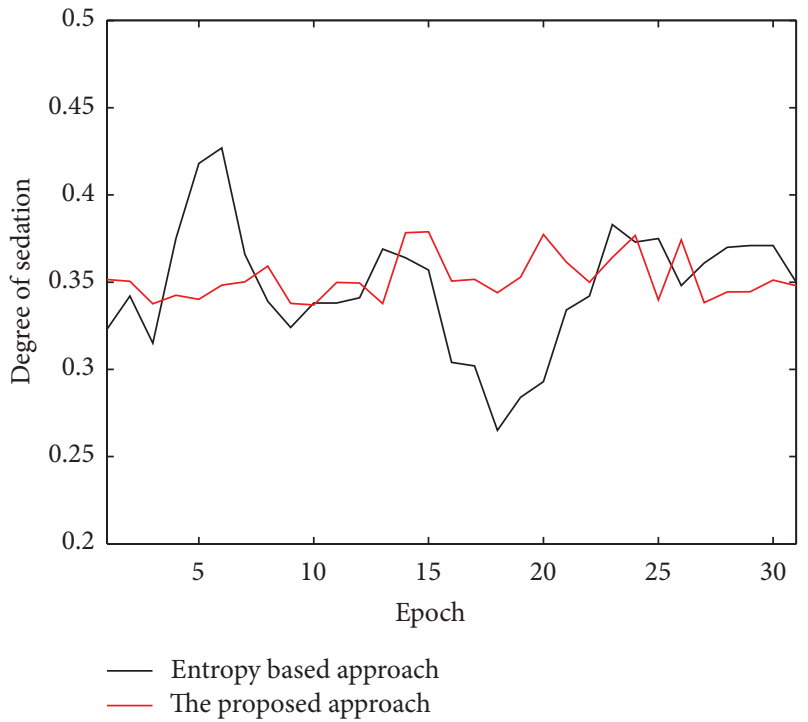

Figure 3: An example of predicted results with 30 epochs.

classified as one group and the others were another group (denoted by $\mathrm{MN}-2$ ). Since the $\mathrm{EtCO}_{2}$ could be used to reflect the status of anesthesia, it was treated as a feature of the status of anesthesia. Therefore, the features including AA-FI, AAET, AA-MAC, $\mathrm{EtCO}_{2}$, and BW are selected as one group and the others were another group (denoted by $\mathrm{MN}-3$ ).

The experimental results expressed as average MSE, maximum MSE, and minimum MSE were shown in Figure 4. The average MSE for ANN, MN-1, MN-2, MN-3, and CRBM were $0.0665,0.0590,0.0551,0.0515$, and 0.0504 , respectively. It is clear that the proposed approach outperforms other approaches. Besides, the performance of modular neural network is better than that of ANN. The MNN can accurately integrate the individual results, which are predicted from biological signals and state of anesthesia. In Figure 4, we also found that the performance variation for ANN is greater than that of other approaches. Therefore, the performance of ANN is closely dependent on the initial weights of a neural network. Using the CRBM, the effect of initial weights of neural network can be greatly reduced.

Comparing the experimental results of $\mathrm{MN}-1, \mathrm{MN}-$ 2 , and $\mathrm{MN}-3$, the performances were quite different for different grouping types. Therefore, the grouping type of features plays an important role in MNN. To achieve an acceptable result, domain knowledge for features could help

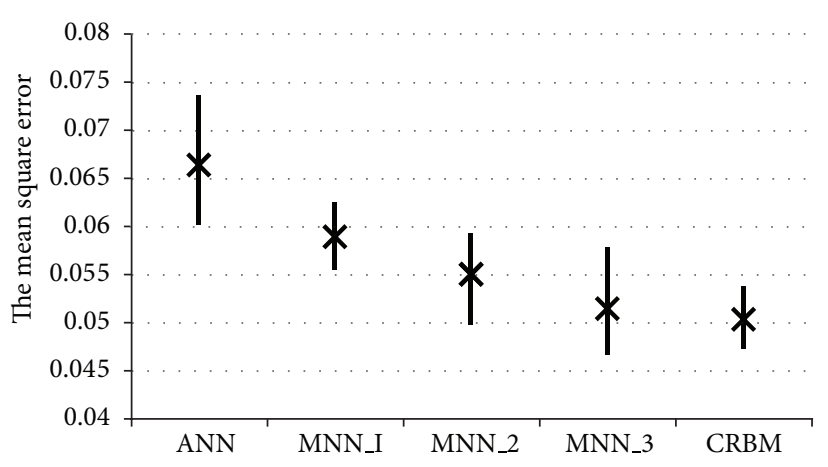

Figure 4: The experimental results of different approaches.

users to design the structure of MMN, but it is difficult. Therefore, CRBM could provide an appropriate approach to automatically map the features into a reasonable space. The experimental results showed that the performance of CRBM is slightly better than that of modular neural network. Thus, it is an effective way to deal with this problem. Moreover, the effect of initial weights can be reduced.

\section{Conclusion}

In this study, a novel approach based on continuous restricted Boltzmann machines is proposed to predict the depth of sedation. The features, which were relative to biological signals and states of anesthesia, successfully represented the characteristics of DOS. Using the CRBM, these features could be effectively mapped to an appropriate domain and then obtained a best performance. Besides, the biological signals, which were used to represent the characteristic of autonomic nervous system, can be individually used to predict the DOS and the results were suitable for many biological systems in clinical practice. The experimental results demonstrated that the proposed approach outperforms feed-forward neural network and modular neural network. Therefore, the proposed approach is able to ease patient monitoring services by using biological systems and promote healthcare quality.

\section{Conflict of Interests}

The authors declare that there is no conflict of interests regarding the publication of this paper. 


\section{Acknowledgment}

The authors would like to thank the Ministry of Science and Technology of Taiwan for financially supporting this research under Contract MOST 103-2221-E-218-009.

\section{References}

[1] M. Neukirchen and P. Kienbaum, "Sympathetic nervous system: evaluation and importance for clinical general anesthesia," Anesthesiology, vol. 109, no. 6, pp. 1113-1131, 2008.

[2] S. G. De Hert, "Volatile anesthetics and cardiac function," Seminars in Cardiothoracic and Vascular Anesthesia, vol. 10, no. 1, pp. 33-42, 2006.

[3] G. Hedenstierna, "Contribution of multiple inert gas elimination technique to pulmonary medicine," Thorax, vol. 50, pp. 8591, 1995.

[4] R. Lenhardt, "The effect of anesthesia on body temperature control," Frontiers in Bioscience, vol. 2, no. 3, pp. 1145-1154, 2010.

[5] G. N. Schmidt, P. Bischoff, T. Standl, M. Issleib, M. Voigt, and J. Schulte am Esch, "ARX-derived auditory evoked potential index and bispectral index during the induction of anesthesia with propofol and remifentanil," Anesthesia and Analgesia, vol. 97, no. 1, pp. 139-144, 2003.

[6] R. A. Rodriguez, L. E. Hall, S. Duggan, and W. M. Splinter, "The bispectral index does not correlate with clinical signs of inhalational anesthesia during sevoflurane induction and arousal in children," Canadian Journal of Anesthesia, vol. 51, no. 5, pp. 472-480, 2004.

[7] H. Litvan, E. W. Jensen, M. Revuelta et al., "Comparison of auditory evoked potentials and the A-line ARX Index for monitoring the hypnotic level during sevoflurane and propofol induction," Acta Anaesthesiologica Scandinavica, vol. 46, no. 3, pp. 245-251, 2002.

[8] B. Bein, "Entropy," Best Practice and Research: Clinical Anaesthesiology, vol. 20, no. 1, pp. 101-109, 2006.

[9] T. Unoki, M. J. Grap, C. N. Sessler et al., "Autonomic nervous system function and depth of sedation in adults receiving mechanical ventilation," The American Journal of Critical Care, vol. 18, no. 1, pp. 42-51, 2009.

[10] J. Y. Lan, M. F. Abbod, R. G. Yeh, S. Z. Fan, and J. S. Shieh, "Intelligent modeling and control in anesthesia," Journal of Medical and Biological Engineering, vol. 32, no. 5, pp. 293-308, 2012.

[11] B. Guignard, "Monitoring analgesia," Best Practice and Research: Clinical Anaesthesiology, vol. 20, no. 1, pp. 161-180, 2006.

[12] S. Kasaoka, T. Nakahara, Y. Kawamura, R. Tsuruta, and T. Maekawa, "Real-time monitoring of heart rate variability in critically ill patients," Journal of Critical Care, vol. 25, no. 2, pp. 313-316, 2010.

[13] X. Li, X. Wang, F. Wang et al., "Measurement of anesthesia depth based on heart rate variability," in Proceedings of the 10th IEEE International Conference on Virtual Environments, HumanComputer Interfaces, and Measurement Systems (VECIMS '12), pp. 186-189, Tianjin, China, July 2012.

[14] A. Nilsson, F. Sjoberg, S. Oster, H. Bek-Jensen, and C. Lennmarken, "Patient-controlled sedation and analgesia with propofol and alfentanil: a preliminary safety evaluation prior to use of non-anaesthesiology doctors," Open Journal of Anesthesiology, vol. 2, pp. 47-52, 2012.
[15] G. Taylor, G. Hinton, and S. T. Roweis, "Modeling human motion using binary latent variables," in Advances in Neural Information Processing Systems, pp. 1345-1352, MIT Press, Cambridge, Mass, USA, 2007.

[16] A.-R. Mohamed and G. Hinton, "Phone recognition using restricted boltzmann machines," in Proceedings of the IEEE International Conference on Acoustics, Speech, and Signal Processing (ICASSP '10), pp. 4354-4357, Dallas, Tex, USA, March 2010.

[17] R. Salakhutdinov, A. Mnih, and G. E. Hinton, "Restricted Boltzmann machines for collaborative filtering," in Proceedings of the 24th International Conference on Machine Learning (ICML '07), pp. 791-798, Oregon, Ore, USA, June 2007.

[18] R. Salakhutdinov and G. Hinton, "Replicated softmax: an undirected topic model," in Advances in Neural Information Processing Systems, vol. 22, 2009.

[19] G. E. Hinton and R. R. Salakhutdinov, "Reducing the dimensionality of data with neural networks," Science, vol. 313, no. 5786, pp. 504-507, 2006.

[20] H. Larochelle and Y. Bengio, "Classification using discriminative restricted boltzmann machines," in Proceedings of the 25th International Conference on Machine Learning (ICML '08), pp. 536-543, July 2008.

[21] A. Coates, H. Lee, and A. Y. Ng, "An analysis of single-layer networks in unsupervised feature learning," in Proceedings of the International Conference on Artificial Intelligence and Statistics, 2011.

[22] A. F. Murray, "Novelty detection using products of simple experts-a potential architecture for embedded systems," Neural Networks, vol. 14, no. 9, pp. 1257-1264, 2001.

[23] H. Chen and A. F. Murray, "Continuous restricted Boltzmann machine with an implementable training algorithm," IEE Proceedings: Vision, Image and Signal Processing, vol. 150, no. 3, pp. 153-159, 2003.

[24] H. Chen, P. C. D. Fleury, and A. F. Murray, "Continuousvalued probabilistic behavior in a VLSI generative model," IEEE Transactions on Neural Networks, vol. 17, no. 3, pp. 755-770, 2006.

[25] J. R. Movellan, P. Mineiro, and R. J. Williams, "A Monte Carlo EM approach for partially observable diffusion processes: theory and applications to neural networks," Neural Computation, vol. 14, no. 7, pp. 1507-1544, 2002.

[26] J. R. Movellan, "A leaming theorem for networks at detailed stochastic equilibrium," Neural Computation, vol. 10, no. 5, pp. 1157-1178, 1998.

[27] J. J. Hopfield, "Neurons with graded response have collective computational properties like those of two-state neurons," Proceedings of the National Academy of Sciences of the United States of America, vol. 81, no. 10, pp. 3088-3092, 1984.

[28] C. H. Yeng, "Using the modular neural network in simulation of the bispectral index for children patients anesthesia depth," Institute of Applied Information, Leader University, 2006. 


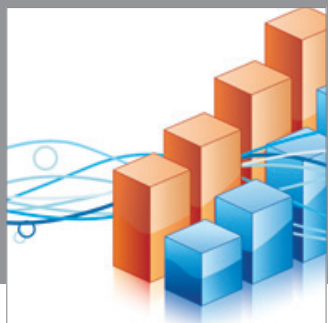

Advances in

Operations Research

mansans

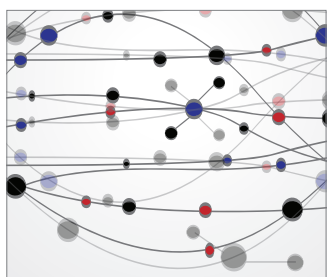

The Scientific World Journal
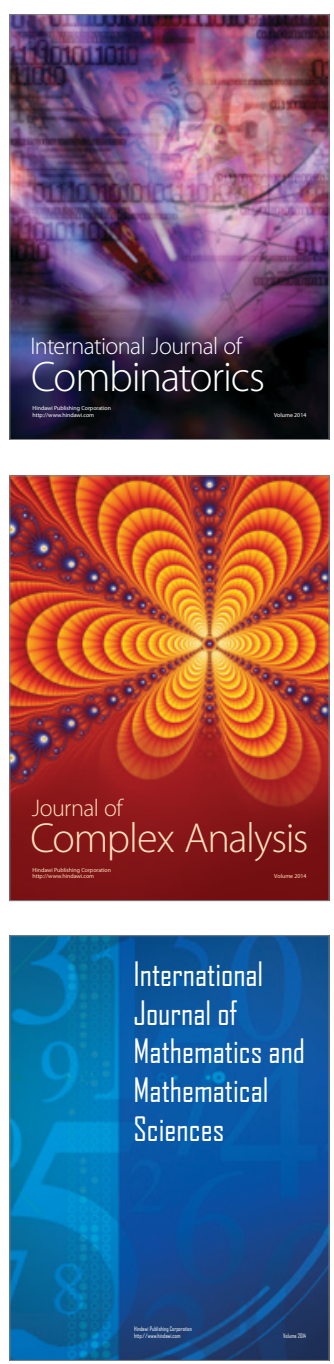
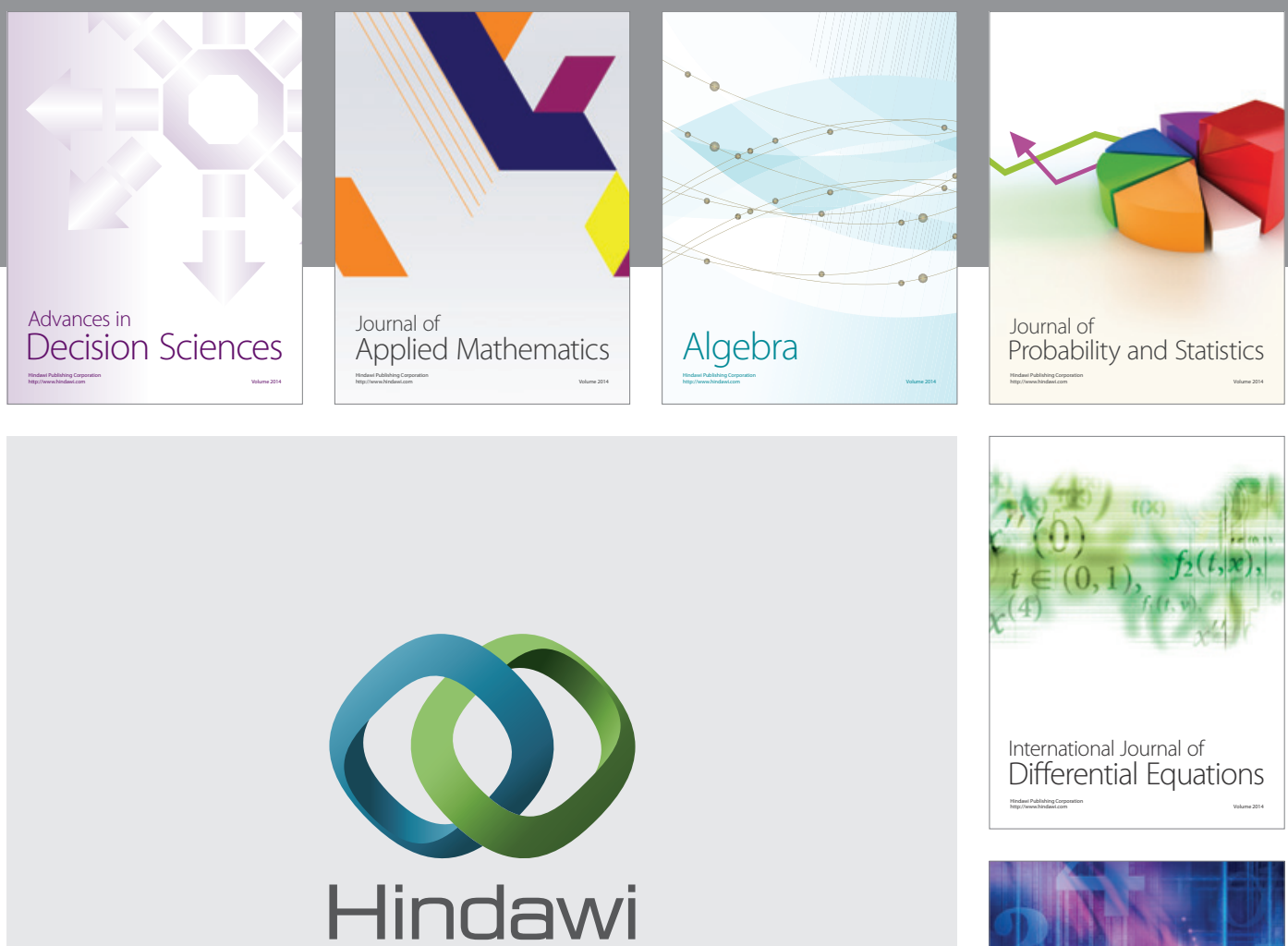

Submit your manuscripts at http://www.hindawi.com
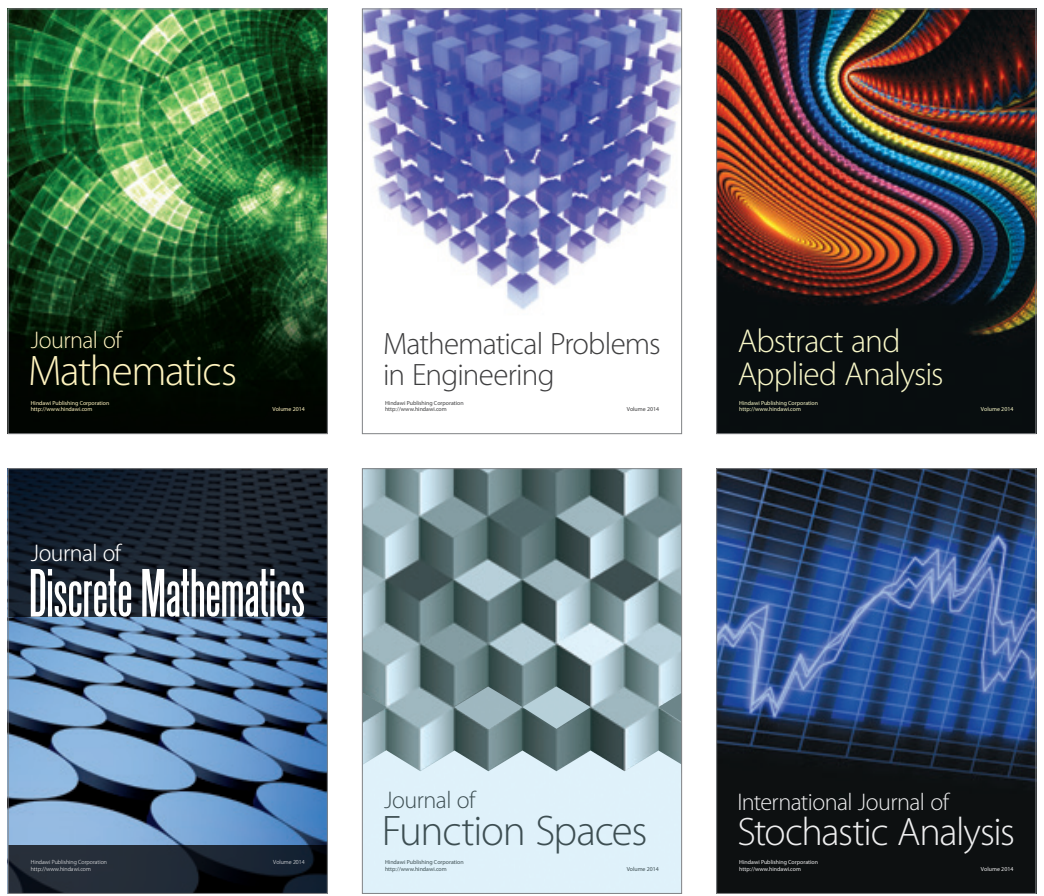

Journal of

Function Spaces

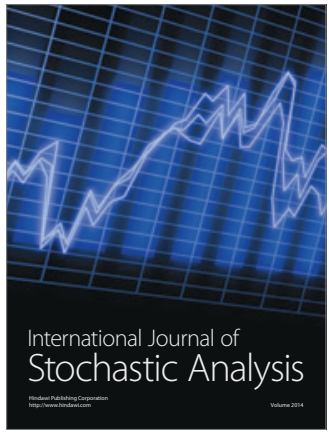

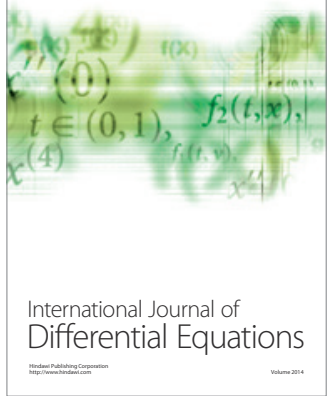
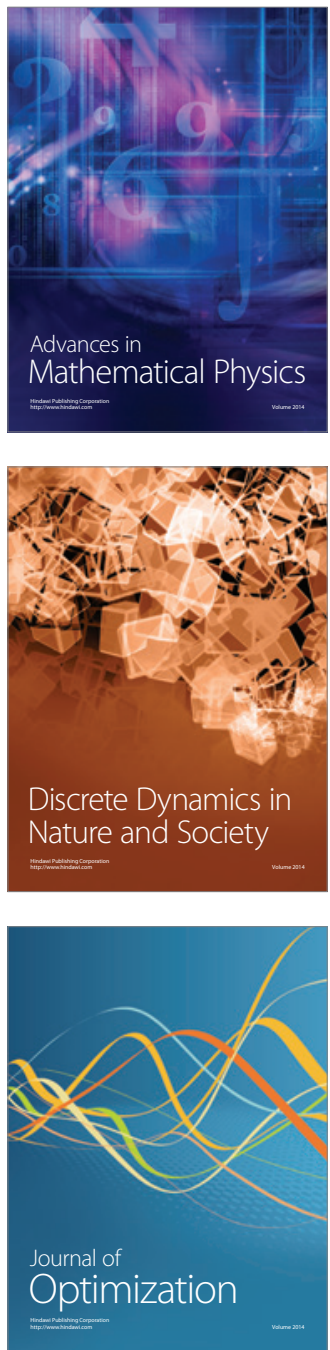